\title{
The Canadian Cardiovascular Society 2018 guideline update for atrial fibrillation - A different perspective
}

Ian G. Stiell, MD, MSc ; M. Sean McMurtry, MD, PhD ${ }^{\dagger}$; Andrew McRae, MD, PhD; Ratika Parkash, MD ${ }^{\S}$; Frank Scheuermeyer, MD, MHSc ${ }^{\pi}$; Clare L. Atzema, MD, MSc ${ }^{* *}$; Allan Skanes, MD $^{\dagger+}$

\section{INTRODUCTION}

In 2018, the Canadian Cardiovascular Society (CCS) published a Focused Update of guidelines for the management of atrial fibrillation (AF). ${ }^{1}$ Some of the updates differ from the Canadian Association of Emergency Physicians (CAEP) 2018 Acute AF Best Practices Checklist. ${ }^{2}$ For emergency department (ED) physicians, the CCS 2018 Update suggested two significant changes: 1) a restriction on which patients can safely undergo cardioversion and 2) an advisory that all acute $\mathrm{AF}$ patients undergoing cardioversion be prescribed four weeks of anticoagulation, regardless of age or comorbidity. Both were "weak recommendation status" based upon "low quality evidence" according to the Grading of Recommendations, Assessment, Development, and Evaluation (GRADE) standards. ${ }^{3}$ These changes were controversial, prompting two additional articles in the Canadian Fournal of Cardiology, one by the leaders of the CCS changes and the other by the authors of this commentary. ${ }^{4,5}$ Here we summarize the main issues as they relate to management of acute AF by ED physicians.

\section{GUIDELINE UPDATE}

The 2018 Update made 18 recommendations, and this review will focus on recommendations 2 and 6. Recommendation 2 updates the prior " 48 -hour rule" that has been recommended by the CCS since 1996, a change driven by several large observational studies from Finland. $^{6,7}$ It now restricts safe cardioversion in non-valvular $\mathrm{AF}$ patients with a $\mathrm{CHADS}_{2}$ score $\geq 2$ to those who present within 12 hours of onset (provided no stroke or transient ischemic attack (TIA) occurred in the past 6 months). Those who have a $\mathrm{CHADS}_{2}$ score $<2$ may be safely cardioverted up to 48 hours from onset.

Recommendation 6 suggests that, regardless of age or comorbidity, all AF patients undergoing cardioversion receive anticoagulation for 4 weeks. Previously, CCS guidelines recommended long-term anticoagulation only for patients at higher risk of stroke (CHADS-65 positive), but CCS panelists placed "greater emphasis on the benefits of stroke prevention compared with the risks of bleeding." The panel acknowledged that "it might be possible to parse these risks either on the basis patient characteristics or the duration of acute AF/AFL," but chose not to do so. ${ }^{1}$

\section{EMERGENCY DEPARTMENT CHALLENGES}

Acute-onset AF contributes to 50,000 annual Canadian ED visits. ${ }^{8}$ Typically, Canadian ED physicians use chemical or electrical cardioversion followed by early discharge home without cardiologist involvement. ${ }^{9,10}$ In 2018, the CAEP formally adapted the prior CCS AF guidelines for ED use. ${ }^{2}$ The CAEP panel included three cardiologists but also sought widespread input from both the cardiology and emergency medicine communities. The CAEP Checklist recommends cardioversion by drug or shock, early ED discharge, and a prescription of oral anticoagulants (OACs) for all patients who are CHADS-65 positive.

From the *Department of Emergency Medicine, Ottawa Hospital Research Institute, University of Ottawa, Ottawa, ON; †Division of Cardiology, University of Alberta, Edmonton, $A B$; $¥$ Departments of Emergency Medicine and Community Health Sciences, University of Calgary, Calgary, $A B$; §Division of Cardiology, Dalhousie University, Halifax, NS; IDepartment of Emergency Medicine, University of British Columbia, Center for Health Evaluation and Outcome Sciences, Vancouver, BC; **Division of Emergency Medicine, University of Toronto, Sunnybrook Health Sciences Centre, and ICES, Toronto, ON; and the ††Division of Cardiology, Western University, London, ON.

Correspondence to: Dr. Ian G. Stiell, Clinical Epidemiology Unit, F657, The Ottawa Hospital, 1053 Carling Avenue, Ottawa, ON K1Y 4E9; Email: istiell@ohri.ca

(C) Canadian Association of Emergency Physicians 
CAEP members are expressing concern about Recommendations 2 and 6 , reporting that they are being advised by cardiology colleagues that these are now "standard of care," despite the designation of a weak recommendation. Recommendation 2 is challenging for ED physicians in its reliance on $\mathrm{CHADS}_{2}$ to guide decision-making on the timing of cardioversion, in contrast with recent encouragement to use the CHADS-65 algorithm. ${ }^{27}$ Although recommendation 3 endorses transesophageal echocardiography to assure safety of urgent cardioversion, this procedure is rarely available on a same-day basis in Canadian hospitals. Recommendation 2, however, is similar to the CAEP Checklist statement, which recommended no cardioversion for patients if onset $\geq 24$ hours and two or more CHADS-65 criteria. Consequently, it is not a substantial practice change for Canadian ED physicians to restrict cardioversion for patients with two or more CHADS- 65 criteria to those presenting within 12 hours of onset.

Recommendation 6, however, represents a large practice change for Canadian ED physicians when managing CHADS-65 negative patients. Given the lack of evidence that OAC reduces the risk of stroke in such low-risk patients, Canadian emergency physicians are concerned at the prospect of prescribing OAC to younger patients. These patients may participate in activities that may lead to head injuries (e.g., contact sports, cycling) and may be at higher risk of bleeding than they are of a stroke. In addition, a prescription of OAC to CHADS-65 negative patients raises other patient-important issues. OAC initiation after spontaneous cardioversion is not mentioned. Warfarin in naive patients may take a considerable time to initiate and stabilize, missing the period of highest risk. In some provinces, novel OAC costs are borne by the patient, particularly for those under age 65. It is challenging to initiate $\mathrm{OAC}$ in the $\mathrm{ED}$, even for patients who are CHADS-65 positive and who need lifelong anticoagulation. ${ }^{11}$ Although presented as a weak recommendation, the suggestion to prescribe a minimum of 4 weeks OAC to all patients carries substantial weight and risks an unwarranted change in practice. Importantly, the GRADE system encourages physicians to apply weak recommendations on a patient-specific basis, considering both clinical factors as well as patient values and preferences.

\section{SUMMARY OF STUDIES}

The standard 48-hour threshold for safe cardioversion, although empirically developed, has been adopted widely into clinical practice. ${ }^{12}$ Seven observational cohort studies and three recent randomized trials (from 2019) were specifically limited to the patients in question (AF duration $\leq 48$ hours) ${ }^{9,13-19}$ Among 4,200 patients, thromboembolic events were rare ( 7 of 4,200 patients, $0.17 \%$ ) after cardioversion for acute AF. Since the 48-hour safety threshold was endorsed by the CCS AF Guideline panel, ${ }^{12,20}$ several large retrospective analyses have challenged this timing. ${ }^{6,7}$ Retrospective analyses of 10,852 cardioversions in 5,441 patients from Finland found that cardioversion in patients with 12-48 hours of symptoms had a higher thromboembolic risk (30 of 2,777 patients, $1.1 \%$ ) compared with cardioversion in patients with less than 12 hours of symptoms ( 8 of 2,440 patients, $0.33 \%$ ). ${ }^{7}$ There was no such timedependent effect for patients who were anticoagulated. ${ }^{21}$

These observational studies also suggest that OAC offers an important reduction in stroke risk for patients undergoing cardioversion for acute $\mathrm{AF} / \mathrm{AFL}^{22}$ in patients with a $\mathrm{CHA}_{2} \mathrm{DS}_{2}-\mathrm{VASc} \geq 2$. This was not demonstrated in those with $\mathrm{CHA}_{2} \mathrm{DS}_{2}$-VASc 0 or 1. Retrospective analyses from two other large registries of patients undergoing cardioversion, each demonstrated higher rates of 30-day thromboembolic events without OAC. ${ }^{23,24}$ Importantly, in one of these studies, there were no events among 4,941 patients in the lowest risk subgroup $\left(\mathrm{CHA}_{2} \mathrm{DS}_{2}\right.$-VASc 0 or 1$)$ and who were not on OAC, a detail not clarified in the other study. A significant limitation was that time of symptom onset prior to cardioversion was not known.

Contemporary prospective data in six Canadian EDs documented only one stroke among 1,091 patients undergoing cardioversion under 48 hours without OAC. ${ }^{9}$ A recent randomized controlled trial compared outcomes among 437 patients undergoing early or delayed cardioversion. OAC use was not prescribed, and only two strokes were recorded at 30 days, one in each arm. ${ }^{19}$ Two other randomized trials, not yet published, followed 480 patients treated in Canadian EDs with cardioversion for 30 days and found no strokes. ${ }^{25,26}$ Cumulatively, these prospective studies have a thromboembolic event rate that is too low to determine the impact of OAC on cardioversion. Nevertheless, the risk of stroke in contemporary practice remains remarkably low. Unfortunately, only retrospective studies are sufficiently large to assess the impact of OAC on stroke rates in the ED. They are limited by not being specifically focused on patients with $\mathrm{AF}<48$ hours duration, or who had OAC initiated at ED discharge as per CCS 
guideline recommendations. For patients at very low stroke risk, there is no direct evidence to support or refute the benefit of short-term OAC in the abovementioned observational studies.

Until large prospective studies can be performed, decision-makers and guideline writers face the dilemma of making a recommendation based on low quality retrospective data or overlooking a signal of potential harm, reassured by a very small number of events in prospective studies. $^{5}$

\section{GRADE WEAK RECOMMENDATION}

The CCS guideline panels use the GRADE system, formal approach guideline development, involving rigorous approaches for grading evidence and for translating summarized evidence into recommendations. ${ }^{3}$ An appeal of GRADE is the ability to quantitate both the quality of evidence (high, moderate, low, or very low, depending upon study limitations) and the strength of the recommendations resulting from such evidence (strong or weak).

To optimally implement weak recommendations into practice, physicians must balance an imperfect set of supporting evidence (with concomitant uncertainty in both treatment effects and treatment hazards for a specific patient) with that patient's individual values and preferences. ${ }^{27}$ This approach is a form of shared decision-making between the physician and the patient. ${ }^{28}$ We must realize that clinical practice guidelines are not a substitute for patient-focused judgement at the bedside. Further, we cannot expect that evidencebased practice will result in uniformity of care as we incorporate uncertainty about risks and benefits or values and preferences into individual clinical decisions. ${ }^{29}$

\section{BOTTOM LINE FOR ED PHYSICIANS}

Box 1 summarizes best practices for who can be safely cardioverted and who requires anticoagulation. All CHADS-65 positive patients clearly require indefinite oral anticoagulation unless absolutely contraindicated. For patients who are CHADS-65 negative, a weak recommendation implies that anticoagulation for 4 weeks is neither mandatory nor the standard of care. Rather this is an opportunity to apply patient-focused clinical judgement. Consideration of longer duration of $\mathrm{AF}$, female sex, presence of vascular disease and other comorbidities, as well as patient preference and values, may encourage oral anticoagulation for 4 weeks after
Box 1. Summary of current recommendations for cardioversion and anticoagulation in acute atrial fibrillation.

1 Cardioversion for non-valvular acute AF patients who are not anticoagulated and have not had a stroke or TIA (in the past 6 months) should be restricted to those who:

a) Present within 12 hours of onset if they have two or more CHADS-65 criteria, unless they can be cleared by transesophageal echocardiography

b) Present within 48 hours of onset if they have 0 or 1 CHADS-65 criteria, unless they can be cleared by transesophageal echocardiography

2 Anticoagulation post-cardioversion:

a) Should be prescribed indefinitely for patients who are CHADS-65 positive

b) Might be considered for a 4-week period for patients who are CHADS-65 negative after careful consideration of risks and benefits and a shared decision-making process with the patient

cardioversion. In the absence of those factors, it may be reasonable to forego OAC. The risk in broad application of oral anticoagulation post-cardioversion is bleeding, especially for those engaged in activities prone to head injury. This must be weighed against the risk of a thromboembolic event post-cardioversion. Fortunately, in CHADS-65 negative patients, both the risk of thromboembolism after cardioversion and the risk of bleeding associated with a simple 4-week course of OAC appear very low. Until there is better evidence to guide us, ED physicians should exercise prudent clinical judgement paired with shared decision-making with their patients.

Keywords: Emergency medicine, cardiac disease, arrhythmia, atrial fibrillation

Competing interests: None declared.

\section{REFERENCES}

1. Andrade JG, Verma A, Mitchell LB, et al. 2018 Focused update of the Canadian Cardiovascular Society guidelines for the management of atrial fibrillation. Can 7 Cardiol 2018;34(11):1371-92. 
2. Stiell IG, Scheuermeyer FX, Vadeboncoeur A, et al. CAEP acute atrial fibrillation/flutter best practices checklist. CFEM 2018;20(3):334-42.

3. Guyatt GH, Oxman AD, Vist GE, et al. GRADE: an emerging consensus on rating quality of evidence and strength of recommendations. BMF 2008;336(7650):924-6.

4. Andrade JG, Mitchell LB. Evidence base for emergency department anticoagulation practices in atrial fibrillation: a conundrum and possible approaches. Can 7 Cardiol 2019; in press.

5. Stiell IG, McMurtry MS, McRae A, et al. Safe cardioversion for patients with acute-onset atrial fibrillation and flutter: practical concerns and considerations. Can 7 Cardiol 2019; in press.

6. Airaksinen KE, Gronberg T, Nuotio I, et al. Thromboembolic complications after cardioversion of acute atrial fibrillation: the FinCV (Finnish CardioVersion) study. $7 \mathrm{Am}$ Coll Cardiol 2013;62(13):1187-92.

7. Nuotio I, Hartikainen JE, Gronberg T, Biancari F, Airaksinen KE. Time to cardioversion for acute atrial fibrillation and thromboembolic complications. FAMA 2014;312 (6):647-9.

8. O'Reilly DJ, Hopkins RB, Healey JS, et al. The burden of atrial fibrillation on the hospital sector in Canada. Can 7 Cardiol 2013;29(2):229-35.

9. Stiell IG, Clement CM, Rowe BH, et al. Outcomes for ED patients with recent-onset atrial fibrillation and flutter (RAFF) treated in Canadian hospitals. Ann Emerg Med 2017;69(5):562-71.

10. Scheuermeyer FX. New frontiers in Canadian atrial fibrillation management. CFEM 2018;20(3):323-4.

11. Scheuermeyer FX, Innes G, Pourvali R, et al. Missed opportunities for appropriate anticoagulation among emergency department patients with uncomplicated atrial fibrillation or flutter. Ann Emerg Med 2013;62(6):557-65.

12. Verma A, Cairns JA, Mitchell LB, et al. 2014 focused update of the Canadian Cardiovascular Society guidelines for the management of atrial fibrillation. Can $\mathcal{f}$ Cardiol 2014;30 (10):1114-30.

13. Weigner MJ, Caulfield TA, Danias PG, Silverman DI, Manning WJ. Risk for clinical thromboembolism associated with conversion to sinus rhythm in patients with atrial fibrillation lasting less than 48 hours. Ann Intern Med 1997;126 (8):615-20.

14. Michael JA, Stiell IG, Agarwal S, Mandavia DP. Cardioversion of paroxysmal atrial fibrillation in the emergency department. Ann Emerg Med 1999;33:379-87.

15. Gallagher MM, Hennessy BJ, Edvardsson N, et al. Embolic complications of direct current cardioversion of atrial arrhythmias: association with low intensity of anticoagulation at the time of cardioversion. 7 Am Coll Cardiol 2002;40 (5):926-33.
16. Burton JH, Vinson DR, Drummond K, et al. Electrical cardioversion of emergency department patients with atrial fibrillation. Ann Emerg Med 2004;44(1):20-30.

17. Stiell IG, Clement CM, Perry JJ, et al. An aggressive protocol for rapid management and discharge of emergency department patients with recent-onset episodes of atrial fibrillation and flutter. CFEM 2010;12(3):181-91.

18. Scheuermeyer FX, Grafstein E, Stenstrom R, et al. Thirtyday outcomes of emergency department patients undergoing electrical cardioversion for atrial fibrillation or flutter. Acad Emerg Med 2010;17(4):408-15.

19. Pluymaekers NAHA, Dudink EAMP, Luermans JGLM, et al. Early or delayed cardioversion in recent-onset atrial fibrillation. N Engl 7 Med 2019;380(16):1499-508.

20. Stiell IG, Macle L. Canadian Cardiovascular Society atrial fibrillation guidelines 2010: management of recent-onset atrial fibrillation and flutter in the emergency department. Can 7 Cardiol 2011;27(1):38-46.

21. Hellman T, Kiviniemi T, Nuotio I, et al. Optimal timing for cardioversion in patients with atrial fibrillation. Clin Cardiol 2018;41(7):966-71.

22. Garg A, Khunger M, Seicean S, Chung MK, Tchou PJ. Incidence of thromboembolic complications within 30 days of electrical cardioversion performed within 48 hours of atrial fibrillation onset. FACC Clin Electrophysiol 2016;2(4): 487-94.

23. Hansen ML, Jepsen RM, Olesen JB, et al. Thromboembolic risk in 16,274 atrial fibrillation patients undergoing direct current cardioversion with and without oral anticoagulant therapy. Europace 2015;17(1):18-23.

24. Sjalander S, Svensson PJ, Friberg L. Atrial fibrillation patients with CHA2DS2-VASc $>1$ benefit from oral anticoagulation prior to cardioversion. Int 7 Cardiol 2016;215:360-3.

25. Scheuermeyer FX, Andolfatto G, Christenson J, Villa-Roel C, Rowe BH. Electrical vs chemical cardioversion in patients with acute atrial fibrillation: a multicenter parallel group randomized controlled clinical trial. Acad Emerg Med 2019; in press.

26. Stiell IG, Perry JJ, Birnie D, et al. A randomized controlled trial of drug vs electrical cardioversion for recent-onset atrial fibrillation. Acad Emerg Med 2019;26[S1], S22.

27. Neumann I, Santesso N, Akl EA, et al. A guide for health professionals to interpret and use recommendations in guidelines developed with the GRADE approach. 7 Clin Epidemiol 2016;72:45-55.

28. Stiggelbout AM, Van der Weijden T, De Wit MP, et al. Shared decision making: really putting patients at the centre of healthcare. BM7 2012;344:e256.

29. Djulbegovic B, Guyatt GH. Evidence-based practice is not synonymous with delivery of uniform health care. $7 A M A$ 2014;312(13):1293-4. 